
\title{
Research S Surare \\ Perspective of Reactive Separation of Levulinic Acid in Conceptual Mixer Settler Reactor
}

\section{Anuj Kumar}

Visvesvaraya National Institute of Technology

Anjali Ingle

Visvesvaraya National Institute of Technology

Diwakar Z. Shende

Visvesvaraya National Institute of Technology

Kailas Wasewar ( $\boldsymbol{\nabla}$ k_wasewar@rediffmail.com )

VNIT Nagpur https://orcid.org/0000-0001-7453-6308

\section{Research Article}

Keywords: Levulinic acid separation, reactive extraction, diffusion coefficient, mixer settler reactor, mass action law

Posted Date: September 8th, 2021

DOI: https://doi.org/10.21203/rs.3.rs-759628/v1

License: (1) (1) This work is licensed under a Creative Commons Attribution 4.0 International License. Read Full License

Version of Record: A version of this preprint was published at Environmental Science and Pollution Research on January 31st, 2022. See the published version at https://doi.org/10.1007/s11356-02218794-y. 


\section{Perspective of Reactive Separation of Levulinic Acid in Conceptual Mixer Settler Reactor}

Anuj Kumar, Anjali Ingle, Diwakar Z. Shende, Kailas Wasewar"

Advanced Separation and Analytical Laboratory (ASAL), Department of Chemical Engineering, Visvesvaraya National Institute of Technology (VNIT), Nagpur 440010, India

*Corresponding author E-mail address: k_wasewar@rediffmail.com

\section{ABSTRACT}

Many industries are using directly biomass as a raw material for the production of valuable biochemicals. These are used different toxic chemicals such as sulphuric acid, hydrochloric acid, nitric acid, etc. as a catalyst during the production of biochemicals and exit downstream waste material and create environmental pollution. Therefore, downstream waste material separation is important for decrease environmental pollution. Levulinic acid is a carboxylic acid which can be present downstream of industrial waste. It is a very important chemical and can be transformed into different important chemicals such as 1-4 pentanediol, aminolevulinic acid, succinic acid, gamma valarolactone, hydoxyvaleric acid, diphenolic acid, etc. It is considered in the top ten important and bio-derived acids. Levulinic acid can be directly produced from biomass through chemical synthesis and fermentation processes at industrial and lab scales. The synthesis process produces the char whereas the fermentation process produces wastes during the production of levulinic acid, thus increasing the production cost and industrial waste downstream. The separation of levulinic acid from the waste is costly and challenging. In this study, reactive extraction was employed with TOA in i-octanol for the separation of levulinic acid. The experimental results have been expressed in various performance parameters like distribution coefficient (0.099-6.14), extraction efficiency (9-86\%), loading ratio (0.09-0.7), and equilibrium constant (11.34-1.05). The mass action law model was also been applied and found the predicted value close with the experimental results. The mixer settler extraction in the series was used to achieve more than $98 \%$ separations of acid. Further, the conceptual approach for levulinic acid separation using mixer-settler reactor scheme is discussed and presented various design parameters including extraction efficiency, diffusion coefficient, extraction equilibrium constant, and loading ratio. This study decreases the industry downstream material and can be solved environmental pollution problems.

Keywords: Levulinic acid separation, reactive extraction, diffusion coefficient, mixer settler reactor, mass action law 


\section{INTRODUCTION}

Many industries exit the downstream wastes during the production of their products. These industrial downstream wastes create soil, water, and air pollution in the environment (Tyagi et al. 2014). Some of the valuable chemicals and biochemicals are also present in the industrial downstream waste and fermentation broth (Shen et al. 2018). The separation of these compounds is important due to their harmful effects on the environment. The various carboxylic acids such as levulinic, lactic, glutaric, aspartic, vanillic, photocatalytic acid, etc. can be carried in the fermentation broth and industrial downstream waste. These acids are very important due to their various applications like fuel, medicine, food, plastics, cosmetics, chemicals, fertilizer, etc.

Levulinic acid (LA) is a carboxylic acid, used in the manufacture of many daily need items. It can be used as a raw material in a variety of industries including plastics, polymers, pharmaceuticals, cosmetics, food, agriculture, biofuel, chemical solvents, rubber manufacturing, etc.(Andersson-Engels et al. 1995; Rackemann and Doherty 2011; Pasquale et al. 2012; Zhang et al. 2012; Mthembu 2015). It can also be used as a platform chemical for the manufacturing of value-added products such as MTHF (methyltetrahydrofuran), a fuel additive; DALA ( $\delta$-aminolevulinic acid), a broad-spectrum herbicide/pesticide; or diphenolic acid (DPA); a substitute for bisphenol A in the polymer industry.

Levulinic acid (LA), also known as keto acid $\left(\mathrm{C}_{5} \mathrm{H}_{8} \mathrm{O}_{3}\right)$, is a ketonic group compound which has largely replaced petroleum-based goods in the chemical and biofuel industries. It is a colorless, small chain fatty acid, soluble in acidic ethanol, diethyl ether, and water. LA is a common substrate in chemical reactions such as condensation, esterification, halogenation, hydrogenation, oxidative dehydrogenation, and salt formation (Zhang et al. 2020). It is a very versatile and potential building block for the synthesis of various organic products (Çelebican et al. 2020). Various fuel and chemical products can be obtained from levulinic acid. Levulinic acid is considered the top twelve organic chemicals by the Energy Department of the USA (Holladay JE, Bozell JJ, White JF 2004). Its permeation enhancers are very safe and effective. Levulinic acid can also be obtained from cellulosic biomass, grass, and wood chips, and has also been regarded as a basic premium material due to its high chemical reactivity. The company GF Biochemicals Ltd. has shared the most levulinic acid manufacture in the world. Hebei Yanuo, Zibo changlin Chemical, Heroy Chemical Industry, Hefei TNJ Chemical, and Langfang Triple well Chemical have started the production of levulinic acid utilising biomass 
as a raw material in recent times. The rice straw, sugarcane bagasse, rice husk, wheat straw, corn straw, and other agricultural wastes contain sucrose, glucose, xylose, and fructose, which can be converted to levulinic acid (Kumar et al. 2019).

According to the industry report, the market of levulinic acid can be predicted to increase at a CAGR of 6\% from 2019 to 2024. The increasing demand for levulinic acid in the plasticizer, pharmaceutical, and cosmetic sectors is the primary factor for its larger market. According to economic analysis, the market of levulinic acid market will be worth USD 71.85 million by 2027, with the growth at a $14.10 \%$ annual rate from 2020 to 2027 .

To remove and recover carboxylic acids from water, a variety of separation techniques have been used including adsorption, extraction, ion exchange, ultrafiltration, nanofiltration, precipitation, electrodialysis, distillation, membrane separation, and reverse osmosis. (Inci and Aydin 2003; Lalikołlu et al. 2015). Among these techniques, extraction (Brouwer et al. 2017), nanofiltration, and electrodialysis (Kim et al. 2013), and adsorption (Datta and Uslu 2017) have been employed in the separation of levulinic acid from aqueous solutions. These processes are expensive, cumbersome, and producing waste material during the process. Some advantages and disadvantages of separation process are listed in Table 1. As a result, the commercial separation of levulinic acid requires low-cost, simple, and environmentally benign process. In comparison to other methods, reactive extraction is a simple, low-cost, promising, and ecologically beneficial method (Antony and Wasewar 2019) because it produces less waste material in the reject stream. The acid molecules present in aqueous phase form complexes with organic phase molecules in reactive extraction, and facilitate improved acid separation.

The purpose of the present study is to separate levulinic acid from industrial downstream material and decreases the problems of environmental pollution. In this study was used promising and environment-friendly method of reactive separation for the recovery of levulinic acid. The partition coefficient $(\mathrm{P})$, distribution coefficient $\left(\mathrm{K}_{\mathrm{D}}\right)$, dimerization constant $(\mathrm{D})$, extraction efficiency $(\eta)$, loading ratio $(Z)$, and experimental equilibrium constant $\left(K_{E}\right)$ have been evaluated by conducting the various experimental runs. The mass action law model was used to predict the various parameters and compared them with experimental values.

\section{MATERIALS AND METHOD}

\subsection{Chemicals}


Levulinic acid ( $\geq 98 \%$ with 1.14 g.cm ${ }^{-3}$ ), i-octanol $\left(\geq 99 \%\right.$ with 0.832 g.cm ${ }^{-3}$ density),

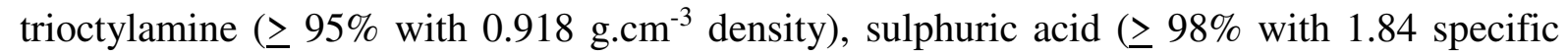
gravity) were purchased from SIGMA-ALDRICH, Loba Chemie Pvt. Ltd., Spectrochem Pvt. Ltd., and Rankem chemicals India respectively and used without the further purification. The details of all chemicals are listed in Table 2.

\subsection{Experimental Procedure}

The experiments were performed using 0.1-1.0 mol. $\mathrm{L}^{-1}$ as the initial concentration of levulinic acid in the aqueous phase because the occurrence of lower range of concentration of acid in the fermentation broths and industrial waste streams. The organic phase was prepared using ioctanol with TOA extractant $(0-80 \% \mathrm{v} / \mathrm{v})$. The sample was taken in equal volume of organic phase and aqueous phase solution in $250 \mathrm{ml}$ conical flask and shaken in an orbital shaker (S24, REMI) for $300 \mathrm{~min}$ at a constant temperature of $298.15 \mathrm{~K}$. After $300 \mathrm{~min}$, sample was taken out and centrifuged for 5 min at $4000 \mathrm{rpm}$ using REMI CENTRIFUGE R-4C for the clear separated phases i.e. the aqueous and organic phase.

\subsection{Analysis}

\subsubsection{HPLC Analysis}

After the phase separation, the aqueous phase was filtered by syringe filter ( 0.45 -micron $)$ and diluted 100 times with double distilled water. The aqueous phase samples were analysed by using an Agilent 1200 HPLC (High-Performance Liquid Chromatography). Sample analysis was carried out using a C-18 column with Refractive Index Detector (RID). The analysis was done using, $5 \mathrm{mM} \mathrm{H}_{2} \mathrm{SO}_{4}$ as the mobile phase, with a flow rate of $0.6 \mathrm{~mL} / \mathrm{min}$ at a column temperature of $35^{\circ} \mathrm{C}$. The HPLC analysis peak is shown in Figure 1.

\subsubsection{Fourier Transform Infrared (FTIR) Analysis}

The spectroscopic techniques used Fourier Transformation Infrared (Shimadzu, IRAffinity-1) were interpreted to interpret the acid: TOA complex production in the organic phase. All measurements were carried out in a potassium bromide window cell. The acid: TOA complexity was observed and examined in levulinic acid reactively extracted with TOA diluted with i-octanol. In the initial aqueous phases, the comparative IR spectrum was obtained and analysed in conjunction with the organic phases of the balance condition concerning stoichiometry in the acid: TOA complex. The vibration peak at $1791 \mathrm{~cm}^{-1}$ represents the $\mathrm{C}=\mathrm{O}$ 
stretching strong carbonyl group (carboxylic acid); $1153 \mathrm{~cm}^{-1}$ and $1053 \mathrm{~cm}^{-1}$ confirm the presence of C-N stretching of amine; and $728 \mathrm{~cm}^{-1}, 1352 \mathrm{~cm}^{-1}, 2866 \mathrm{~cm}^{-1}$, and $2922 \mathrm{~cm}^{-1}$ confirmed the C-H stretching of alkanes group. FTIR spectra of range $500-4000 \mathrm{~cm}^{-1}$ are shown in Figure 2.

\section{RESULTS AND DISCUSSION}

After the production of levulinic acid through the fermentation broth and synthesis process, it occurs in a lower and very dilute range of concentration in the waste streams. Therefore, the sample of levulinic acid was prepared with $0.1-1 \mathrm{~mol}^{-1} \mathrm{~L}^{-1}$ as initial concentration of acid in the aqueous phase. The organic phase containing TOA as reactive solvent and i-octanol as diluent was used.

\subsection{Physical Extraction}

Between the organic and aqueous phases, the distribution of un-dissociated LA molecules are characterised as follows:

$$
[L A]_{a q} \stackrel{P}{\leftrightarrow}[L A]_{\text {org }}
$$

In this situation, the partition coefficient $(\mathrm{P})$ can be defined as:

$$
P=\frac{[L A]_{o r g}}{[L A]_{a q}}
$$

The dimerization constant of LA can be represented as:

$$
2[L A]_{\text {org }} \stackrel{D}{\leftrightarrow}[L A]_{2 . \text { org }}
$$

$$
D=\frac{[L A]_{2 . o r g}}{\left[L A_{\text {org }}\right]^{2}}
$$

Where $[L A]_{2 . o r g}$ and $\left[L A_{\text {org }}\right]^{2}$ are the concentration of levulinic acid in the organic phase and dimerization form respectively.

The overall distribution coefficient is influenced by the concentration of $\mathrm{H}^{+}$ions in the solution as well as its ionic strength. Overall distribution coefficient can be written as:

$$
K_{D}=\frac{[L A]_{\text {org }}^{\text {total }}}{[L A]_{a q}^{\text {total }}}=\frac{[L A]_{\text {org }}+2\left[L A_{2}\right]_{\text {org }}}{[L A]_{a q}+\left[L A^{-}\right]}=\frac{P+2 P^{2} D[H L A]_{a q}}{1+K_{L A} /\left[L^{+}\right]_{a q}}
$$

In the present study, the dilute concentration of levulinic acid $\left(0.1-1\right.$ mol. $\left.\mathrm{L}^{-1}\right)$ was used. Therefore, the term $K_{L A} /\left[L^{+}\right]_{a q}$ in denominator of (5) can be considered as negligible. Hence, equation (5) can be considered as:

$$
K_{D}=P+2 P^{2} D[L A]_{a q}
$$


Alternatively, equation (6) can be written as:

$$
[L A]_{\text {org }}=P[L A]_{a q}+2 P^{2} D[L A]_{a q}^{2}
$$

The physical extraction and its results are discussed in form of distribution coefficient (0.0990.681), dimerization coefficient (226), partition coefficient (0.051), and extraction efficiency (9-40.5\%) respectively. The experiments were performed using initial concentrations (0.1-1 mol. $\mathrm{L}^{-1}$ ) of levulinic acid with i-octanol, and maximum extraction efficiency $40.5 \%$ with 0.681 distribution coefficient was observed at equilibrium condition in case of physical equilibrium. The values of the dimerization coefficient (D) and partition coefficient $(\mathrm{P})$ initiated the linear regression method using equation 7.

\subsection{Chemical Extraction}

The physical extraction efficiency is not sufficient for the separation of levulinic acid separation from the industrial downstream waste. To improve $\mathrm{K}_{\mathrm{D}}$ values, low-density nonreactive solvents can be used as diluents along with reactive solvents. During the experimental work, trioctylamine was used in organic phase. The trioctylamine (TOA) is less soluble in water and provides a higher separation efficiency of the acid. In TOA, the N-H bond carries more polarity than the $\mathrm{C}-\mathrm{H}$ bond but is lesser than the $\mathrm{O}-\mathrm{H}$ bond. On comparing the physical properties of amines, the polarity becomes apparent. Therefore, TOA transferred more molecules of acid from aqueous phase to organic phase and improve the extraction efficiency. The equilibrium isotherms of acid concentration between aqueous and organic phases are shown in Figure 3.

The values of distribution coefficients were determined in the range of 0.47-6.14 with (20-80 vol \%) of TOA. The distribution coefficient was found to be decreased with increasing the levulinic acid concentration in aqueous phase. The higher $\mathrm{K}_{\mathrm{D}}$ value was obtained at lower concentration of levulinic acid $\left(0.1 \mathrm{~mol} . \mathrm{L}^{-1}\right)$. The higher concentration of acid leads to increase the loading ratio in organic phase and the back extraction may be performed for the recovery and regeneration of TOA and levulinic acid. More number of molecules interacted in the extractant phase of chemical extraction results in better mass transfer from the aqueous phase. Figure 3 depicts the chemical equilibria of the aqueous and organic phases. The acid-amine complexes were formed due to the strong interaction between amine and acid that results in high distribution coefficients (Yang et al. 1991). Based on the analysis of $K_{D}$ values, TOA was 
found to be a good reactive component in i-octanol for the separation of LA from the aqueous phase.

The extraction efficiency $(\eta)$ was estimated in the range of 32-86\%, using TOA with i-octanol. In case of chemical extraction, the extraction efficiency increased with the TOA concentration up to $40 \%$ TOA in i-octanol and therefore it decreases with the further increase of TOA (Figure 4). It may be attributed to the governing synergistic effect of hydrogen bonding and polarity of i-octanol with acid molecules (Wasewar et al. 2004). The separation efficiency depends on the physical properties of diluent like solubility, molecular weight, dielectric constant, refractive index, etc(Kumar et al. 2020). The i-octanol was used along with reactive solvents, which leads to the higher separation efficiency of LA.

The loading ratio is defined as the ratio of acid concentration in the organic phase at equilibrium to the initial concentration of reactive solvent in the organic phase. The loading ratio $(\mathrm{Z})$ was determined to analyses the complex formation in reactive extraction. The values of $\mathrm{Z}$ were found in the range of $0.06-0.7$ using $20-80 \%$ TOA in i-octanol. The $\mathrm{Z}$ values depend on the strength of the acid-extractant interaction and the stoichiometry of the overall extraction equilibrium (Kertes and King 1986; Keshav et al. 2009; Wasewar et al. 2011). The loading ratio decreases with the increase of reactive solvent concentration in the organic phase that enhances a more favorable solvating agent (Yang et al. 1991). The loading fraction was found to be greater than 0.5 , hence the acid-extractant complex of $1: 1$ and $2: 1$ in the organic phase can be formed.

The equilibrium constant, $\mathrm{K}_{\mathrm{E}}$ is affected by the formation of complexes between levulinic acid and the reactive solvent. It depends on the loading ratio; i.e., the complex can form (1:1) or (2:1) in the organic phase (Figure 5). The following equation can be used to calculate the equilibrium complexation constant; $\mathrm{K}_{\mathrm{E}}$ :

$$
\begin{array}{lr}
\frac{Z}{1-Z}=K_{E}[L A]_{a q} & \text { If } Z<0.5 \\
\frac{Z}{2-Z}=K_{E}[L A]_{a q}^{2} & \text { If } Z>0.5
\end{array}
$$

The complexation extraction equilibrium constant $\left(\mathrm{K}_{\mathrm{E}}\right)$ was found in the range of (11.34-1.05) with (0.457-1.83 mol.L $\left.\mathrm{L}^{-1}\right)$ TOA in i-octanol. The various estimated value of $\mathrm{K}_{\mathrm{E}}$ is listed in Table 3. 
213 The reaction mechanism can be predicted in the organic phase wherein $n$ molecules of TOA

214 per molecule of levulinic acid take part in the reaction to form the acid-extractant complex, 215 given as:

216

$$
L A+n \overline{T O A} \leftrightarrow \overline{T B P_{n} L A}
$$

217 Theoretically, the mass action law can be used to estimate the equilibrium extraction constant $218\left(\mathrm{~K}_{\mathrm{E}}\right)$ and the number of extractant molecules per acid molecule (n). As per the definition of complexation extraction equilibrium constant, $\mathrm{K}_{\mathrm{E}}$ can be written as:

$$
K_{E}=\frac{\overline{\left[T O A_{n} L A\right]}}{[L A]{\overline{[T O A]^{n}}}^{n}}
$$

221 Also, levulinic acid dissociates at equilibrium in the aqueous phase. It can be written as:

222

$$
L A \leftrightarrow L^{+}+A^{-}
$$

Where $K_{a}$, is the dissociation constant

$\mathrm{C}_{\mathrm{LA}}$ can be expressed as:

226

$$
K_{a}=\frac{\left[L^{+}\right]\left[A^{-}\right]}{[L A]}
$$

227

228

$$
C_{L A}=[L A]\left[A^{-}\right]
$$

Using equations (13) and (14)

$$
[L A]=\frac{C_{L A}}{\left(1+\frac{K_{a}}{\left[L^{+}\right]}\right)}
$$

229 The distribution coefficient and extraction efficiency can be determined using equations (16) 230 and (17);

231

$$
\begin{gathered}
K_{D}=\frac{\bar{C}_{L A}}{C_{L A}}=\frac{\overline{\left[T O A_{n} L A\right]}}{C_{L A}} \\
\eta \%=\left(\frac{K_{D}}{1+K_{D}}\right) \times 100
\end{gathered}
$$




$$
K_{D}=\frac{K_{E}[\overline{T O A}]^{n}}{\left(1+\frac{K_{a}}{\left[L^{+}\right]}\right)}
$$

On taking the logarithm on both sides and rewriting the equation:

$$
\begin{aligned}
& \log K_{D}+\log \left(1+\frac{K_{a}}{\left[L^{+}\right]}\right)=\log K_{E}+n \log ([\overline{T O A}]) \\
& \log \left(\frac{K_{D}}{\left(1+\frac{K a}{\left[L^{+}\right]}\right)}\right)=\log K_{E}+n \log ([\overline{T O A}])
\end{aligned}
$$

$$
\text { Where }[\overline{T O A}]=\overline{[T O A}_{i n}-n\left[\overline{T O A_{n} L A}\right]
$$

Assuming $\overline{[T O A]}_{i n} \gg n\left[\overline{T O A_{n} L A}\right]$, and the initial concentration $\overline{[T O A}_{\text {in }}$ can be substituted in equation (20) to determine the value of $\mathrm{n}$ and $K_{E}$.

$\mathrm{Eq}^{\mathrm{n}}(20)$ represents a straight line if plotted $\log K_{D}+\log \left(1+\frac{K_{a}}{\left[L^{+}\right]}\right)$vs $\log \left([\overline{T O A}]_{i n}\right)$. The value of $K_{E}$ and $n$ can be found using the intercepts and the slope of a linear line (Figure 6).

The values of $n$ and $K_{E}$ were calculated with the help of plot between $\log K_{D}+\log \left(1+K_{L A}\right.$ $\left./\left[\mathrm{L}^{+}\right]\right)$vs $\log [\mathrm{TOA}]$ in which yields a straight line with a slope of $\mathrm{n}$ and an intercept of $\log \mathrm{K}_{\mathrm{E}}$. The graphical representation can be used to calculate the values of $\mathrm{K}_{\mathrm{E}}$ and $\mathrm{n}$ for various extraction systems. The $K_{E}$ value defines the separation of acid. If $K_{E}<1$ means product separation efficiency is low as compared to the reactant. Whereas if $\mathrm{K}_{\mathrm{E}}>1$, it defines the good separation efficiency of the acid. The values of $K_{E}$ and $n$ were found (12.40-0.99) and (0.350.99 ) with different concentrations of TOA and levulinic acid.

The experimental value of $\mathrm{K}_{\mathrm{E}}$ is closed to the predicted value by mass action law value. Therefore, the mass action law holds good model represents the understanding of the separation of levulinic acid.

\section{Mixer Settler Reactor}

\subsection{Mass balance for multiple reactor}

According to the experimental results and its understanding, the process needs process intensification-based designing. The mixer settler reactor can be used in series to increase the separation efficiency by more than $98 \%$ and decrease the concentration in waste stream. In this 
study, the best and optimum experimental data was used for design the conceptual mixer settler reactor in series and achieving $98 \%$ recovery of levulinic acid.

The diagram of stage-to-stage calculation is shown in Fig. 7. The given values of inlet concentrations of levulinic acid $[L A]_{\text {inlet } 1}$ and $[L A]_{\text {inlet } 2}$ for reactor first and second. The outlet value defined as Mass balance for mixer settler reactor can be derived as:

$$
[L A]_{\text {org outlet } 1}=[L A]_{\text {inlet } 1}-[L A]_{\text {aq outlet } 1}
$$

The calculation for the first mixer settler reactor-

$$
[L A]_{\text {aq outlet } 1}=[L A]_{\text {inlet } 1}-\left(\text { Efficiency } \times[L A]_{\text {inlet } 1}\right)
$$

The calculation for the first mixer settler reactor-

$$
\begin{aligned}
& {[L A]_{\text {aq outlet } 1}=0.1-(0.86 \times 0.1)} \\
& {[L A]_{\text {aq outlet } 1}=0.014 \mathrm{~mol} . L^{-1}}
\end{aligned}
$$

Calculation of second mixer settler reactor-

In this case first reactor aqueous outlet consider inlet for second reactor, then

$$
\begin{aligned}
{[L A]_{a q} } & =0.014-(0.86 \times 0.014) \\
{[L A]_{a q} } & =0.00196 \mathrm{~mol} . L^{-1}
\end{aligned}
$$

Then overall efficiency: 
284

Many industries were used toxic acid as a catalyst $\left(\mathrm{H}_{2} \mathrm{SO}_{4}, \mathrm{HCl}, \mathrm{HNO}_{3}\right.$, etc. $)$ for the manufacturing of valuable levulinic acid. During the production of acid, the primary effect of the chemical industry on the environment and the lifestyle as well, is due to the pollution, arising from the industrial activities, resulting in wastewater polluting, gas emissions, underground water and natural ponds, etc. The industrial downstream material required the separation method for controlling these types environmental pollution. The mixer settler reactor designing can be play an important role for preventing the environmental pollution.

\section{CONCLUSION}

The main obstacles in dealing with industrial waste and fermentation broth aqueous streams can be addressed using the reactive extraction method. The experimental data suggested that reactive separation is an effective and flexible process for recovering the valuable levulinic acid as compared to the other methods like membrane, adsorption, distillation, etc. The overall results conclude the highest separation efficiency as $86 \%$ at $40 \%$ TOA with initial concentration $\left(0.1 \mathrm{~mol} . \mathrm{L}^{-1}\right)$ of levulinic acid. The lower concentration of TOA provides sufficient extraction efficiency. The mass action law applied to experimental results and found the predicted value near to experimental value. Therefore, mass action law can be applied to the experimental results as the predicated values are close found to experimental value. Therefore, mass action law can represent a model for the separation of levulinic acid. To increasing the separation efficiency, the mixer settler reactor was designed in series and could achieve the recovery of levulinic acid as $98 \%$. Moreover, the conceptual mixer settler reactor method carries with zero waste and with minimum energy usage during the recovery process of levulinic acid. This experimental study can be used to decrease industrial waste material and environmental pollution.

\section{- Ethical Approval}

Authors are approval that this article presented original work and did not submit in other journals.

\section{- Consent to Participate}

Authors are giving consent to participate

\section{- Consent to Publish}

Authors are giving consent to publish the present work as per publisher policy.

\section{- Authors Contributions}


Anuj Kumar: Conceived and designed the experiments; Performed the experiments; Analysed and interpreted the data; wrote the paper.

Anjali Ingle: Analysed the HPLC analysis and interpreted the data

Diwakar Z. Shende: Conceived and designed the experiments; Analyzed and interpreted the data; Contributed reagents, materials, analysis tools, or data.

Kailas Wasewar: Conceived and designed the experiments; Analyzed and interpreted the data; Contributed reagents, materials, analysis tools, or data.

\section{- Funding}

Not applicable

\section{- Competing Interests}

Not applicable

- Availability of data and materials

Data and other materials will be made available on request to the authors.

\section{REFERENCES}

Andersson-Engels S, Berg R, Svanberg K, Svanberg S (1995) Multi-colour fluorescence imaging in connection with photodynamic therapy of $\delta$-amino levulinic acid (ALA) sensitised skin malignancies. Bioimaging 3:134-143. https://doi.org/10.1002/13616374(199509)3:3<134::AID-BIO4>3.3.CO;2-T

Antony FM, Wasewar K (2019) Reactive extraction: a promising approach to separate protocatechuic acid. Environmental Science and Pollution Research. https://doi.org/10.1007/s11356-019-06094-x

Brouwer T, Blahusiak M, Babic K, Schuur B (2017) Reactive extraction and recovery of levulinic acid, formic acid and furfural from aqueous solutions containing sulphuric acid. Separation and Purification Technology 185:186-195. https://doi.org/10.1016/j.seppur.2017.05.036

Çelebican Ö, İnci İ, Baylan N (2020) Investigation of adsorption properties of levulinic acid by a nanotechnological material. Journal of Molecular Structure 1203:. https://doi.org/10.1016/j.molstruc.2019.127454

Datta D, Uslu H (2017) Adsorption of levulinic acid from aqueous solution by Amberlite XAD4. Journal of Molecular Liquids 234:330-334. https://doi.org/10.1016/j.molliq.2017.03.084

Holladay JE, Bozell JJ, White JF JD (2004) Top value added chemicals from biomass volume I - Results of screening for potential candidates from sugars and synthesis gas. II: 
Inci I, Aydin A (2003) Extraction of hydroxycarboxylic acids with MIBK/Toluene solutions of amines. Journal of Scientific and Industrial Research 62:926-930

Kertes AS, King CJ (1986) Extraction chemistry of fermentation product carboxylic acids. Biotechnology and Bioengineering 28:269-282. https://doi.org/10.1002/bit.260280217

Keshav A, Wasewar KL, Chand S (2009) Reactive extraction of propionic acid using tri-noctylamine, tri-n-butyl phosphate and aliquat 336 in sunflower oil as diluent. Journal of Chemical Technology and Biotechnology 84:484-489. https://doi.org/10.1002/jctb.2066

Kim JH, Na JG, Yang JW, Chang YK (2013) Separation of galactose, 5-hydroxymethylfurfural and levulinic acid in acid hydrolysate of agarose by nanofiltration and electrodialysis. Bioresource Technology 140:64-72. https://doi.org/10.1016/j.biortech.2013.04.068

Kumar A, Shende DZ, Wasewar KL (2019) Production of levulinic acid: A promising building block material for pharmaceutical and food industry. Materials Today: Proceedings 29:790-793. https://doi.org/10.1016/j.matpr.2020.04.749

Kumar A, Shende DZ, Wasewar KL (2020) Separation of Levulinic Acid by Reaction with Tri- n-butylphosphate Diluted in Nontoxic Solvents. Journal of Chemical and Engineering Data 65:3002-3007. https://doi.org/10.1021/acs.jced.0c00007

Lalikołlu M, Gök A, Gök MK, Aşçl YS (2015) Investigation of Lactic Acid Separation by Layered Double Hydroxide: Equilibrium, Kinetics, and Thermodynamics. Journal of Chemical and Engineering Data 60:3159-3165. https://doi.org/10.1021/acs.jced.5b00324

Mthembu LD (2015) Production of Levulinic Acid From Sugarcane Bagasse. 26-27

Pasquale G, Vázquez P, Romanelli G, Baronetti G (2012) Catalytic upgrading of levulinic acid to ethyl levulinate using reusable silica-included Wells-Dawson heteropolyacid as catalyst. Catalysis Communications $18: 115-120$. https://doi.org/10.1016/j.catcom.2011.12.004

Rackemann DW, Doherty WO (2011) The conversion of lignocellulosics to levulinic acid. Biofuels, Bioproducts and Biorefining 5:198-214. https://doi.org/10.1002/bbb.267

Shen Q, Chen Y, Lin H, et al (2018) Agro-industrial waste recycling by Trichosporon fermentans: conversion of waste sweetpotato vines alone into lipid. Environmental Science and Pollution Research 25:8793-8799. https://doi.org/10.1007/s11356-018$1231-\mathrm{Z}$

Tyagi VK, Lo SL, Rajpal A (2014) Chemically coupled microwave and ultrasonic prehydrolysis of pulp and paper mill waste-activated sludge: Effect on sludge solubilisation and anaerobic digestion. Environmental Science and Pollution Research 21:6205-6217. https://doi.org/10.1007/s11356-013-2426-y

Wasewar KL, Shende D, Keshav A (2011) Reactive extraction of itaconic acid using tri-n-butyl phosphate and aliquat 336 in sunflower oil as a non-toxic diluent. Journal of Chemical Technology and Biotechnology 86:319-323. https://doi.org/10.1002/jctb.2500 
Wasewar KL, Yawalkar AA, Moulijn JA, Pangarkar VG (2004) Fermentation of glucose to lactic acid coupled with reactive extraction: A review. Industrial and Engineering Chemistry Research 43:5969-5982. https://doi.org/10.1021/ie049963n

Yang ST, White SA, Hsu ST (1991) Extraction of Carboxylic Acids with Tertiary and Quaternary Amines: Effect of $\mathrm{pH}$. Industrial and Engineering Chemistry Research 30:1335-1342. https://doi.org/10.1021/ie00054a040

Zhang J, Wu S bin, Li B, Zhang HD (2012) Advances in the catalytic production of valuable levulinic acid derivatives. ChemCatChem 4:1230-1237. https://doi.org/10.1002/cctc.201200113

Zhang Z, Wu Y, Gao L, Xiao G (2020) Pervaporation separation of levulinic acid aqueous solution by ZSM-5/PDMS composite membrane. Journal of Applied Polymer Science 138:1-10. https://doi.org/10.1002/app.49611

1 
Table 1 Some advantages and disadvantages of various methods of separation

\begin{tabular}{lll}
\hline Method & Advantages & Disadvantages \\
\hline Distillation & -Simple installation & -High energy required \\
& -Products of high purity & -High cost \\
& -Low capital investment & -Components are thermally stable \\
& -Low risk & \\
\hline Membrane & -Product of high yield & -Membrane fouling \\
& -Low energy required & \\
& -Reliable & \\
\hline Evaporation & -Low capital investment, & -High energy demand \\
& -Low-risk operation & \\
\hline Adsorption & -Easy to install & -High costs and energy demand \\
& - Relatively high & \\
& Selectivity & \\
\hline Reactive extraction & -Control of the pH in the reactor & \\
& - Solvents can be used & \\
& -Higher product concentration & \\
\hline
\end{tabular}


Table 2 The physiochemical properties of various chemicals used

\begin{tabular}{llcccc}
\hline \multicolumn{1}{c}{ Chemical } & \multicolumn{1}{c}{ Supplier } & Purity\% & IUPAC name & Viscosity/cP at 25 C $^{\circ}$ & Density/g.cm $^{-3}$ \\
\hline Levulinic acid & SIGMA-ALDRICH & 98 & 4-oxopentanoic acid & - & 1.14 \\
i-octanol & Loba Chemie Pvt. & 99 & 2- ethylhexanol & 7.36 & 0.832 \\
& Ltd. India & & & 7.862 & 0.8 \\
Trioctylamine & Spectrochem Pvt. & 95 & Tricaprylamine & & \\
(TOA) & Ltd. Mumbai. India & & & 26.7 & 1.83 \\
Sulphuric acid & Rankem chemicals & 98 & - & \\
\hline
\end{tabular}

407

408 
409 Table 3 Define extraction equilibrium constant of experimental and mass action law

\begin{tabular}{ccc}
\hline TOA $\left(\mathbf{m o l}_{\mathbf{L}} \mathbf{L}^{\mathbf{1}}\right)$ & Experimental $\left(\mathbf{K}_{\mathbf{E}}\right)$ & Mass action law $\left(\mathbf{K}_{\mathbf{E}}\right)$ \\
\hline 0.457 & 11.34 & 12.40 \\
0.915 & 7.41 & 6.45 \\
1.372 & 2.91 & 3.56 \\
1.83 & 1.05 & 0.99 \\
\hline
\end{tabular}

410

411 


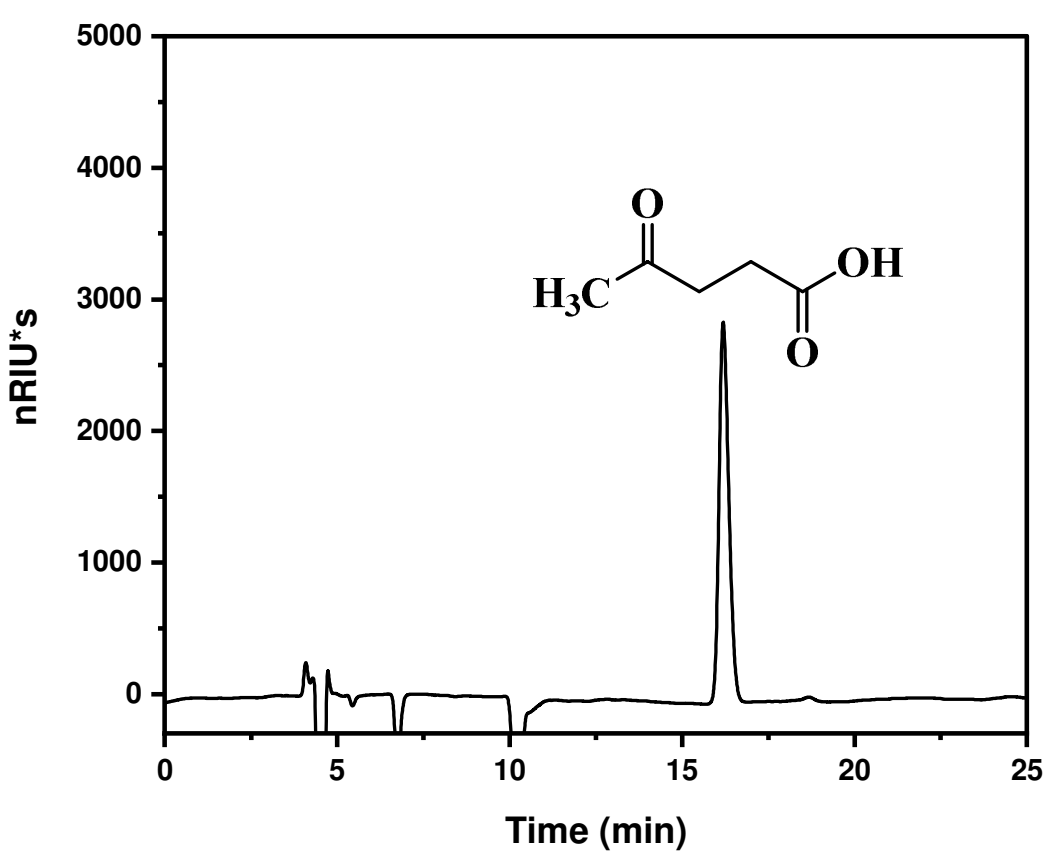






428

Figure 2 FTIR Spectra (500-4000 $\mathrm{cm}^{-1}$ range) for levulinic acid

430 


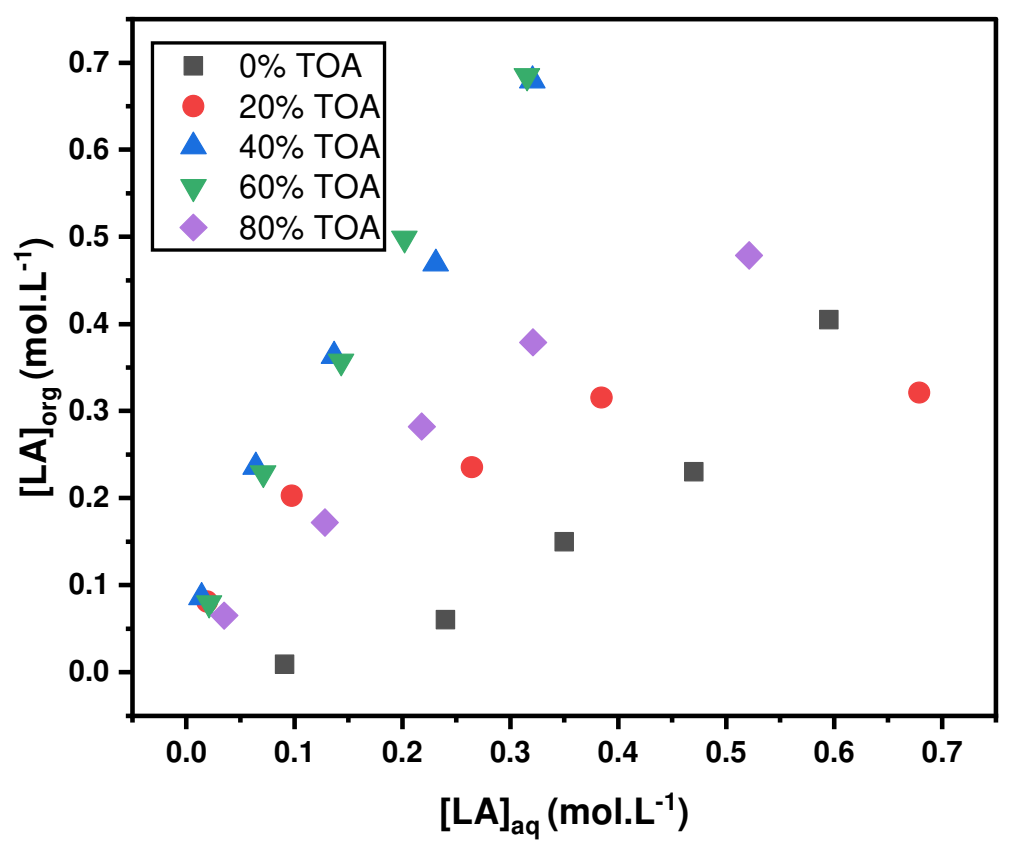

432

Figure 3 Equilibrium isotherms of acid concentration between aqueous and organic phase with TOA $(0-80$ $\mathrm{v} / \mathrm{v} \%)$ in i-octanol. 


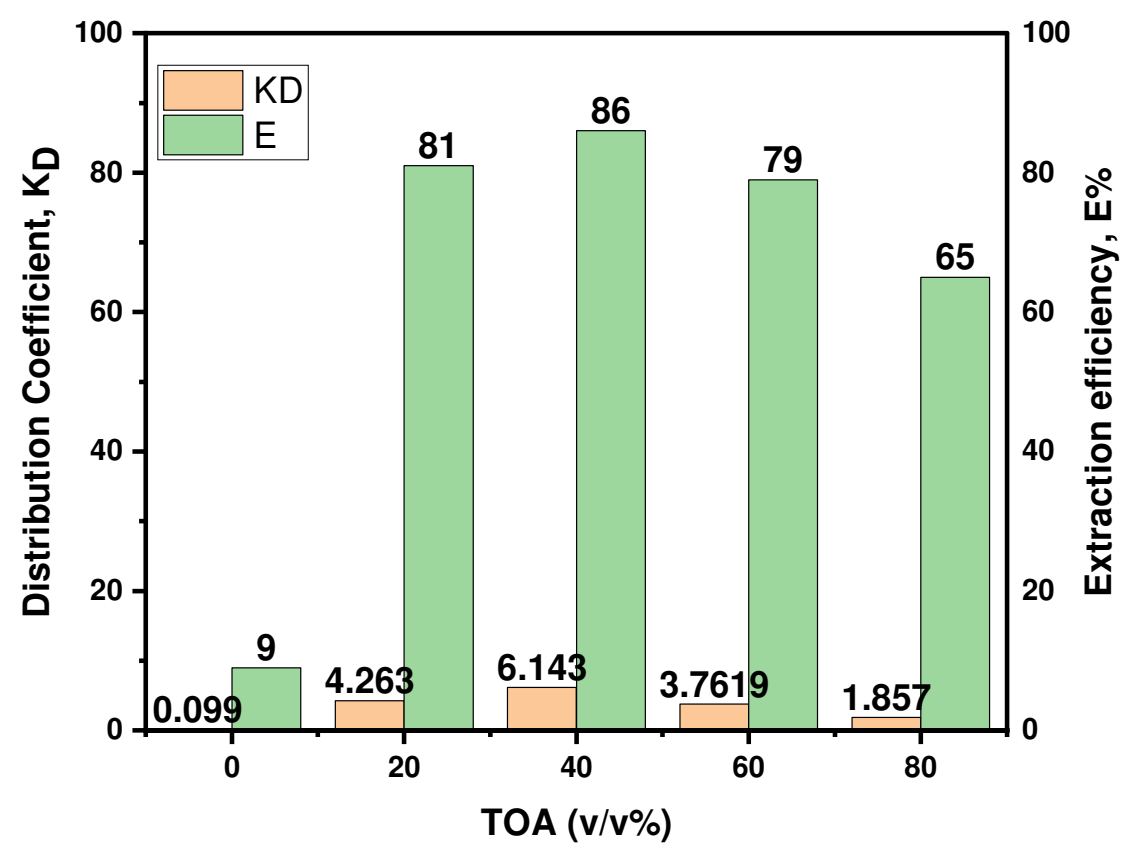

437 TOA varies in i-octanol

440

441

442 

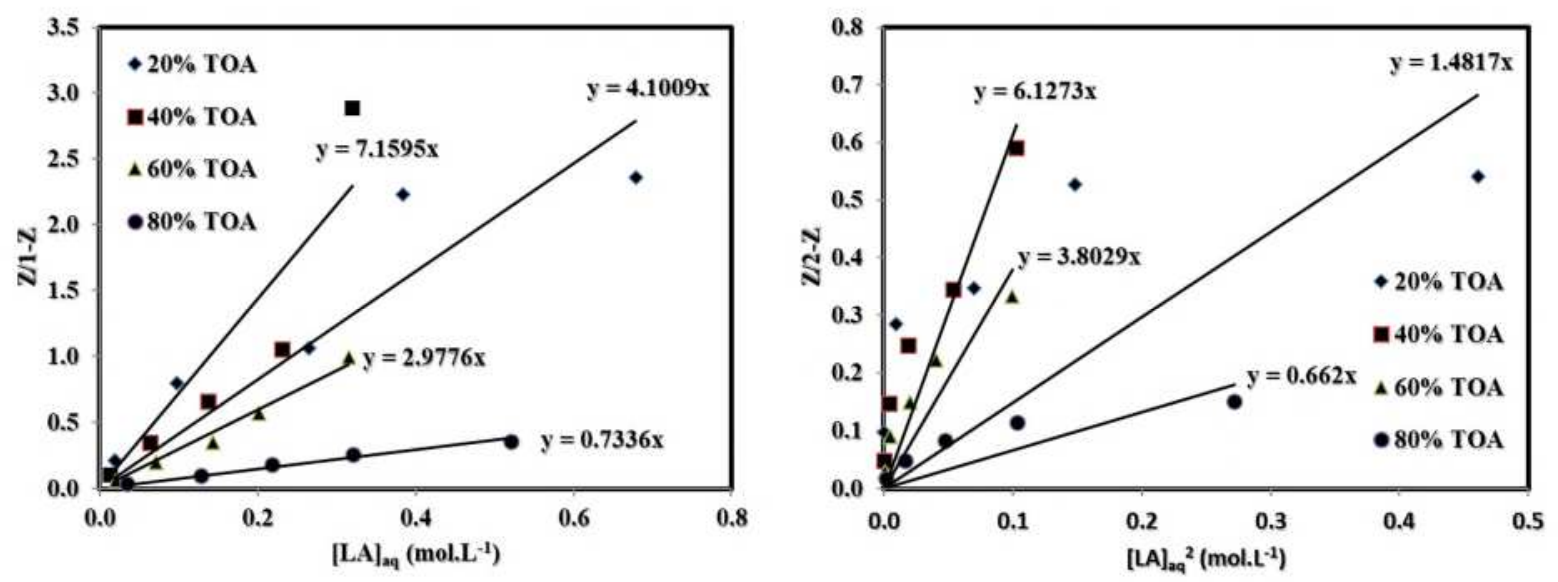

444

445

Figure 5 Plot of $\mathrm{Z} /(1-\mathrm{Z})$ vs $[\mathrm{LA}]_{\mathrm{aq}}$ and $\mathrm{Z} /(2-\mathrm{Z})$ vs $[\mathrm{LA}]_{\mathrm{aq}}{ }^{2}$ for the estimation of $(1: 1)$ and $(2: 1)$ levulinic acidTOA equilibrium complexation constant in i-octanol. 

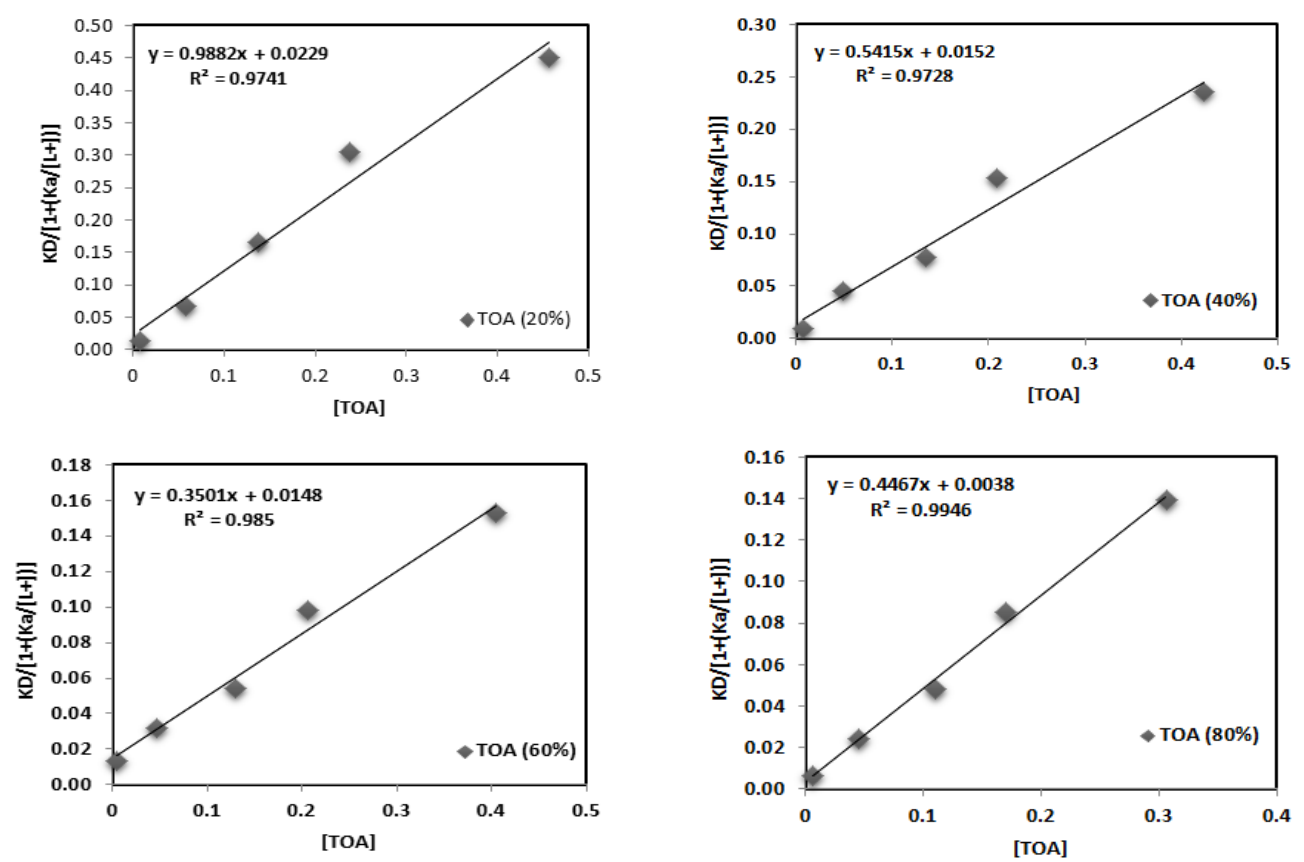

449 Figure 6 Variation of $\boldsymbol{K}_{D} /\left[\mathbf{1}+\left(\boldsymbol{K}_{\boldsymbol{a}} /{ }_{\left[\boldsymbol{L}^{+}\right]}\right)\right]$with various TOA concentrations for the various concentration of 450 levulinic acid 


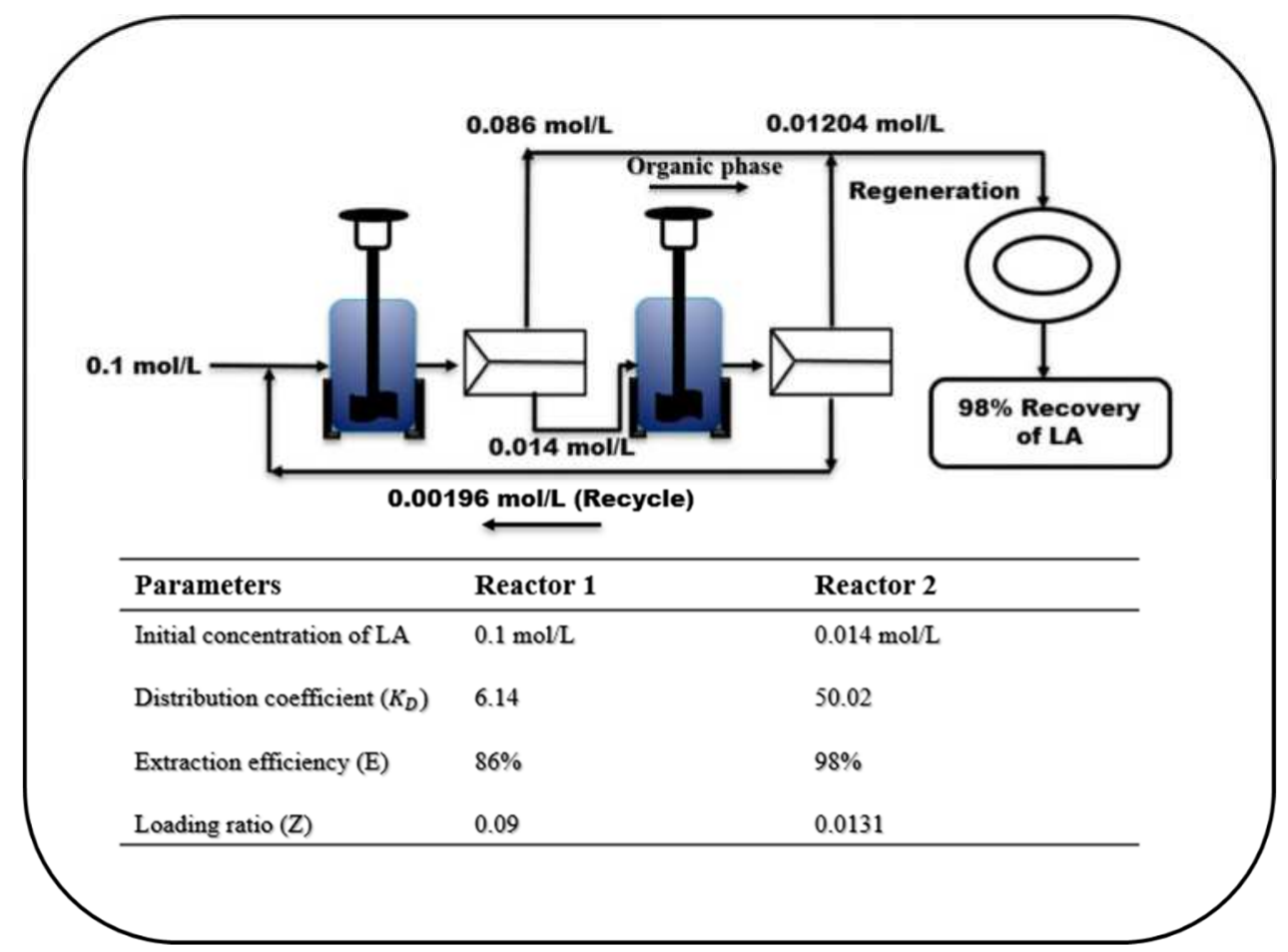

Figure 7 Conceptual mixer settler reactor connected in series for achieving 98\% recovery of LA. 